\title{
EFECTO DEL GRITO COMO UN RECURSO ERGOGÉNICO PSICOLÓGICO EN PRACTICANTES DE KUNG FU
}

\author{
Juan Diego Zamora Salas y Walter Salazar Rojas \\ Escuela de Educación Física y Deportes \\ Universidad de Costa Rica, San José, Costa Rica \\ E-mail: juandiegozamora@hotmail.com
}

\begin{abstract}
Resumen
Zamora Salas, J. D., y Salazar Rojas, W. (2002). Efecto del grito como un recurso ergogénico psicológico en practicantes de Kung Fu. Revista de Ciencias del Ejercicio y la Salud, 2(2), 32-37. El presente estudio analizó el efecto del grito como mecanismo inhibidor de sensaciones como el dolor y la fatiga en practicantes de Kung Fu, utilizando una prueba de push ups. Se formaron tres grupos de forma aleatoria, cada uno conformado por $n=10$, cuyas edades estaban comprendidas entre los 15 y 30 años. Los sujetos del Grupo Experimental sin grito y Grupo Experimental con grito, les correspondía realizar las prácticas de push ups aplicando los tratamientos correspondientes durante cinco semanas; mientras que los sujetos del grupo control no realizaban éstas prácticas durante el período establecido, sólo les correspondía el pre-test y post-test. A los valores recolectados durante las pruebas se les aplicó una Anova 3 x 2 y un análisis posthoc de la interacción mostrando la existencia de una diferencia significativa entre el pre-test y el post-test tanto en el grupo que aplicó el grito personal como en el grupo que no lo aplicó, lo cual no se manifestó entre el pre-test y post-test del grupo control. Al comparar los datos obtenidos del grupo que utilizó el grito personal con respecto al grupo que no utilizó el grito como tratamiento, se encontró una diferencia significativa entre ambos métodos. Con el estudio se concluyó que el grito en combinación con el ejercicio físico permite observar incrementos significativos en la cantidad de repeticiones de un ejercicio de fuerza para el tren superior como son las push ups, luego de cinco semanas de tratamiento. PALABRAS CLAVES: Grito, ergogénico, psicológico, artes marciales, Kung Fu.
\end{abstract}

\section{INTRODUCCIÓN}

Desde hace varios años investigadores, científicos, entrenadores, etc.; se han dado a la tarea de estudiar e indagar nuevas formas de mejorar el rendimiento en determinadas actividades y así remover las barreras fisiológicas, mecánicas y psicológicas que producen una disminución en el rendimiento deportivo.

Una de las principales barreras que se presentan en el rendimiento deportivo es la fatiga muscular, la cual es definida como la incapacidad del músculo de mantener una contracción o serie de contracciones a un determinado nivel, además de generar sensaciones de incomodidad o de dolor (Aragón y Fernández, 1995).
El fenómeno de la fatiga no es únicamente de origen muscular, sino también puede ser de origen nervioso por lo que se utiliza el término de fatiga neuromuscular (Aragón y Fernández, 1995).

En un estudio realizado por Ikai y Steinhaus (1961), se utilizaron diferentes métodos como la hipnosis, sonidos fuertes y el grito (personal) para inhibir el dolor y la fatiga producidos por factores psicológicos; mejorando así el rendimiento y la fuerza muscular.

El estudio realizado por Ikai y Steinhaus (1961); consistió en medir la tensión de los músculos flexores del antebrazo derecho en un esfuerzo máximo utilizando un cable tensiómetro, para lo cual los sujetos debían gritar tan fuerte como fuera posible mientras ejercían el jalón final. 
Se comprobó un aumento del 12,2\% de fuerza en tensión, en comparación al ejercicio sin gritar, cuyo aumento altamente significativo fue atribuible al grito (Ikai y Steinhaus, 1961).

Según Ikai y Steinhaus (1961), el estímulo del grito aumentó la fuerza por encima de los niveles normales debido a una modificación temporal de la función del sistema nervioso central. Ellos afirman que las personas operan normalmente a un nivel de inhibición neural que les impide expresar su verdadera capacidad de fuerza y que ésta capacidad se establece en gran parte por el tipo de fibra que predomina en el músculo y por la colocación mecánica del hueso y del músculo.

(1961), las experiencias pasadas desagradables relacionadas con el ejercicio, un ambiente familiar demasiado protector o el temor a lesionarse, son algunos factores por los cuales las personas normalmente son incapaces de expresar su capacidad de máximo esfuerzo, generando una inhibición neuromuscular.

En el estudio realizado por Ikai y Steinhaus (1961) se concluye que la utilización del grito es suficiente solamente para bloquear los procesos inhibitorios internos en un sujeto y así llegar a un rendimiento aparentemente supermáximo; afirmando también que éste grito o ruido personal como el fuerte llamado de "Alah" utilizado por los levantadores de pesas árabes y el grito de batalla frecuentemente asociado con el avance de fuerzas armadas, hace ser el mecanismo bloqueador de inhibiciones internas, permitiendo ganar competencias y que en la zona de batalla transforma a los cobardes en héroes.

Éste grito muchas veces es utilizado en diferentes actividades deportivas como en el levantamiento de pesas (Ikai y Steinhaus, 1961), en competencias de campo en el atletismo y muy frecuentemente por los practicantes de artes marciales en sus combates, formas y rompimientos.
Otro importante estudio fue el realizado por Morales, Owen y O`Conneli (1999), en donde se investigó si una desinhibición vocal producida por un gruñido incrementaba la fuerza en un levantamiento con pesas. Se le colocaron a los sujetos electrodos en el músculo bíceps femoral y así poder llevar a cabo una electromiografía durante el levantamiento que ellos efectuaran, con el fin de conocer la producción de fuerza en éstos músculos en el momento en que los sujetos realizaban la destreza sin gruñir y con el gruñido.

Al finalizar el estudio Morales y col. (1999), no encontraron una diferencia significativa en la producción de fuerza que podía generar el músculo bíceps femoral en el momento en que el sujeto realizaba el levantamiento aplicando el gruñido y en aquel en donde no aplicaba el gruñido. El estudio concluyó, que la desinhibición vocal a través del gruñido no parece incrementar significativamente la producción de fuerza durante la actividad de un grupo muscular grande como fue el bíceps femoral.

Es conocido por muchas personas que en los diferentes estilos de las artes marciales, sus practicantes utilizan el grito personal en el momento en el cual efectúan alguna destreza o técnica, tales como golpes, patadas, rompimientos entre otros. En un estudio realizado por Zamora y Salazar (2002) con practicantes de artes marciales, se estudió el grito personal en una prueba de salto largo sin impulso; mostrando una diferencia significativa entre el grupo que utilizó el grito en el momento del salto con respecto al grupo que no utilizó éste tratamiento; en donde el grupo que utilizó el grito aumentó la distancia del salto.

En su estudio Zamora y Salazar (2002), concluyen que la implementación del grito personal como un recurso ergogénico psicológico puede afectar positivamente alguna destreza específica, si éste es utilizado en el preciso momento en el que se realiza la destreza.

Sin embargo aún no se conoce con exactitud los efectos que tiene el grito en el 
rendimiento físico, especialmente en los practicantes de artes marciales, por lo que surge el interés y la necesidad de investigar cómo el grito personal puede afectar ya sea positiva o negativamente el desempeño de los artistas marciales.

El objetivo del presente estudio es conocer el efecto que tiene el grito personal en combinación con el ejercicio físico con respecto a la cantidad de repeticiones de un ejercicio específico.

\section{METODOLOGIA}

\section{Sujetos}

En el estudio participaron 30 sujetos varones practicantes de Kung $\mathrm{Fu}$, cuyas edades estaban comprendidas entre los 15 y 30 años.

Se formaron tres grupos de forma aleatoria, cada uno conformado por un $n=10$ sujetos. El tratamiento del Grupo 1 o Experimental sin grito consistía en no aplicar el grito personal durante las prácticas; al Grupo 2 o Experimental con grito el tratamiento correspondiente consistía en aplicar un grito personal durante las prácticas y el Grupo 3 o Control no realizarían las prácticas durante el estudio.

\section{Instrumentos de medición}

En el presente estudio se propone como prueba un test de push ups (conocidas también como lagartijas o pechadas); mientras que las denominadas prácticas consisten en la ejecución del mismo ejercicio con la implementación de los tratamientos.

\section{Procedimientos}

La destreza o ejercicio de las push ups fue explicada y demostrada a los diferentes sujetos de los grupos tanto el control como los experimentales, aún conociendo que los sujetos de los diferentes grupos conocían y tenían experiencia en la destreza. Antes de iniciar el estudio se les aplicó un pre-test y al finalizar el estudio un post-test, de la misma destreza; en donde ningún grupo aplicaría el grito y así poder comprobar si hubo un cambio o mejora del rendimiento en la cantidad de push ups, posterior a la implementación de los tratamientos durante el período en que se llevó a cabo el estudio.

\section{Figura 1. Posición correcta para la ejecución de las push ups.}

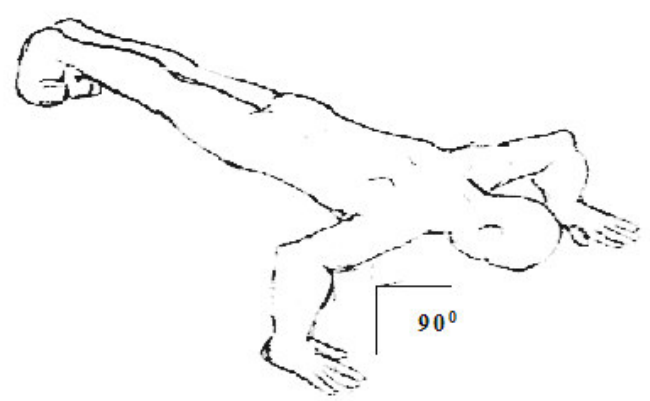

El estudio se llevó a cabo durante cinco semanas, en las cuales los sujetos del grupo sin grito y del grupo con grito debían de realizar las prácticas de push ups dos veces a la semana, en donde mantenían los mismos días para realizar las prácticas durante todo el estudio. Los sujetos no podían realizar las push ups ningún otro día de la semana.

Antes de iniciar los test o las prácticas de push ups, los sujetos de los diferentes grupos realizaban de forma breve el mismo calentamiento y estiramiento de brazos para evitar alguna lesión.

Tanto las pruebas como las prácticas de push ups, consistían en llegar a un esfuerzo máximo. En el caso de los test, cuando los sujetos lograban el esfuerzo máximo y éstos perdían la posición correcta o caían al suelo producto del dolor o fatiga muscular se consideraba finalizada la prueba.

En el caso de las prácticas, el grupo sin grito realizaba la destreza hasta lograr el esfuerzo máximo. Cuando sus integrantes 
caían al suelo, producto del dolor o la fatiga muscular debían continuar inmediatamente con el ejercicio sin que hubiera una fase de descanso o de relajación. Se consideraba como finalizada la práctica para éste grupo en el momento en que volvían a caer al suelo o perdían la posición correcta del ejercicio.

El grupo con grito realizaba la misma práctica y cuando sus integrantes caían al suelo producto del dolor o la fatiga muscular, debían de gritar lo más fuerte posible y proseguir con el ejercicio sin que hubiera una fase de descanso o de relajación. Se consideraba como finalizada la práctica en el momento en que volvían a caer al suelo o perdían la posición correcta del ejercicio.

Como los dos grupos experimentales se encontraban en el mismo momento de la práctica, los integrantes de un grupo ejecutaban la práctica, mientras que los otros sujetos se colocaban a la par con el brazo extendido debajo del pecho de éste para contar las push ups y verificar el momento de la caída. Luego de que todos los sujetos de un grupo experimental finalizaban la práctica se cambiaban y continuaba el siguiente grupo experimental.

La señal de iniciar la práctica o prueba (test) era dada al mismo tiempo por el administrador de la práctica o prueba (test) a todos los sujetos que conformaban un grupo experimental, por lo que debían estar posicionados correctamente y con el cuerpo en alto, como se muestra en la Figura 1.

\section{Análisis estadístico}

Se determinó la estadística descriptiva: promedio (M) y desviación estándar (DS). También se utilizó un análisis de varianza (ANOVA) de dos vías, grupos (3 niveles) por mediciones (2 niveles) y un análisis de seguimiento de efectos simples.

\section{RESULTADOS}

En la Tabla No. 1 se muestran los valores para los promedios y sus respectivas desviaciones estándar (DS) obtenidos en el pre-test y post-test de la prueba de push ups, tanto para el grupo control como para los grupos experimentales; donde se puede apreciar que los valores más altos se presentan en el grupo experimental con grito.

Tabla 1. Estadística descriptiva de la cantidad de push ups obtenidos en el Pre-test y Post-test del Grupo Control y de los Grupos Experimentales

\begin{tabular}{lllllll}
\hline \multirow{2}{*}{ Grupos } & \multicolumn{3}{c}{ Pre-test } & \multicolumn{3}{c}{ Post-test } \\
\cline { 2 - 7 } & M & & DS & M & \multicolumn{1}{c}{ DS } \\
\hline Control & 23.5 & \pm & 7.14 & 25 & \pm & 6.85 \\
Sin grito & 28.1 & \pm & 7.61 & 34.4 & \pm & 7.99 \\
Con grito & 32.6 & \pm & 9.53 & 42.6 & \pm & 12.98 \\
\hline
\end{tabular}

Los resultados del análisis de varianza de dos vías (ANOVA $3 \times 2$ ), se muestran en la Tabla No. 2; donde la $\mathrm{F}=6.70$ demuestra una diferencia significativa entre los grupos de sujetos. La F= 19.68 refleja una diferencia significativa entre el Pre-test y el Post-test, observándose un aumento en el número de repeticiones de push ups en el post-test. La F=3.39 muestra una interacción significativa entre los tratamientos y las mediciones.

Tabla 2. Resultados de la Anova 3x2.

\begin{tabular}{lcc}
\hline Fuentes de Varianza & F & $\boldsymbol{\omega}^{2}$ \\
\hline Grupos & $6.70 *$ & $25.68 \%$ \\
Mediciones & $19.68 *$ & $7.33 \%$ \\
Interacción & $3.39 *$ & $1.87 \%$ \\
\hline
\end{tabular}

$* \mathrm{p}<0.05$

Luego de realizarse el respectivo análisis de varianza de dos vías se prosiguió con un análisis de la interacción, el cual mostró una diferencia significativa en el post-test del grupo con grito y el grupo sin grito, con respecto al post-test del grupo control. Además, el grupo que aplicó el grito obtuvo puntajes promedio significativamente mayores que el grupo sin grito.

En la Figura 2, se aprecia el efecto de la interacción de manera que el mayor incremento entre el pre-test y el post-test, se 
encontró en el grupo experimental que utilizó el grito como tratamiento.

\section{Figura 2. Comparación del efecto de gritar o no gritar en la cantidad de repeticiones de push ups.}

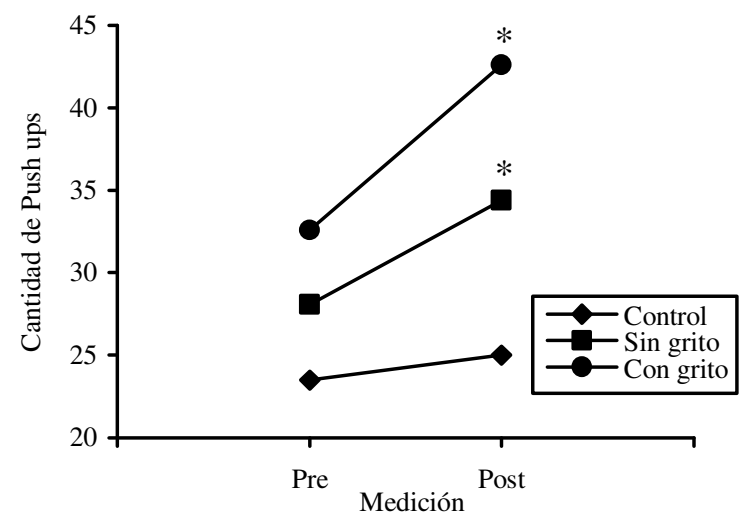

$* \mathrm{p}<0.05$, todos los grupos difieren entre sí en el post test.

Los resultados obtenidos luego de llevar a cabo los diferentes análisis estadísticos muestran la existencia de una diferencia significativa entre el pre-test y el post-test tanto en el grupo que aplicó el grito personal como en el grupo que no aplicó el grito, lo cual no se manifestó entre el pre-test y post-test del grupo control.

\section{DISCUSION}

Al comparar los datos obtenidos del grupo que utilizó el grito personal con respecto al grupo que no utilizó el grito como tratamiento, se encontró una diferencia significativa entre ambos métodos; en donde el grupo que utilizó el grito personal logró aumentar la capacidad de continuar con el ejercicio de las push ups y de llevar a cabo esfuerzos mayores; luego de que los sujetos caían al suelo o en el momento en que perdían la posición correcta producto de la fatiga muscular, la cual según afirma Aragón y Fernández (1995), no le permite a un sujeto mantener una serie de contracciones a un determinado nivel; además de la aparición de las sensaciones de incomodidad o de dolor que están asociadas con la fatiga muscular.

Ikai y Steinhaus (1961) afirman en su estudio que el grito permite aumentar el número de repeticiones en un ejercicio y de realizar esfuerzos casi supermáximos, debido a la inhibición de las sensaciones que se le presentan al sujeto durante un ejercicio prolongado como son el dolor y la fatiga.

Con el estudio se puede afirmar que el grito puede ser un importante mecanismo o recurso psicológico bloqueador momentáneo de diversas sensaciones producidas por algún ejercicio o actividad física, permitiendo al sujeto o deportista aumentar levemente el umbral del dolor, o sobreponerse al dolor con la utilización de éste, y así poder continuar con el ejercicio o la actividad física; logrando llevar a cabo esfuerzos mayores a su capacidad como lo afirman Ikai y Steinhaus (1961).

También el grito puede ser utilizado o aplicado como un recurso ergogénico legal no sólo en las artes marciales, sino también en aquellas actividades donde puede aparecer rápidamente sensaciones que inhiben la capacidad del sujeto de continuar con una determinada acción, o en aquellas en donde el sujeto necesita realizar un esfuerzo mayor a su capacidad como en los levantamientos de pesas o en las pruebas de campo en el atletismo.

Finalmente se concluye con los resultados del presente estudio, que el grito personal en combinación con ejercicio físico permite incrementos significativos en la cantidad de repeticiones de un ejercicio de fuerza para el tren superior como son las push ups, luego de cinco semanas de tratamiento.

\section{REFERENCIAS}

Aragón, L. y Fernández, A. (1995). Fisiología del Ejercicio. Respuestas, entrenamiento y medición. Costa Rica, San José: Universidad de Costa Rica.

Ikai, M.y Steinhaus, A. (1961). Some factors modifying the expression of human strength. Journal of Applied Physiology, 16, 157-163. 
Revista de Ciencias del Ejercicio y la Salud ${ }^{\circledR}$

Vol. 2, $N^{o} 2,2002$

Morales, Z.; Owen, S. y O`Conneli, D. (1999). Vocal Disinhibition (grunting) does not increase dead lift force in college athletes or nonathletes. Perceptual and Motor Skills, 89, 233- 234.
Zamora, J., y Salazar, W. (2002). Efecto del grito como recurso ergogénico psicológico en la potencia de piernas en practicantes de Kung Fu. Cuadernos de Psicología del Deporte, 2 (1), 57-66. 